\title{
SOME OPERATORS ON UNIFORM SPACES
}

\author{
BY \\ SEYMOUR GINSBURG AND J. R. ISBELL(1)
}

Introduction. The principal results in this paper concern a class of uniform spaces defined by the condition that every uniformly locally uniform covering is uniform. Such spaces, called locally fine spaces, generalize the fine spaces, i.e. spaces bearing the finest uniformity consistent with the topology. The most striking results are (i) that, subject to a mild cardinality restriction, the completion of a locally fine space is determined by its uniformly continuous real-valued functions (generalization of Shirota's theorem [14] for fine spaces), and (ii) that every real-valued uniformly continuous function on a subspace of a locally fine space can be extended (generalization of a known result for fine spaces). However, both of these results are trivial for subspaces of fine spaces (i.e. the step from fine spaces to subspaces is trivial), and we have not been able to determine whether this includes all the locally fine spaces. The principal value of the concept "locally fine" is that it is a simply defined uniform property which distinguishes a well-behaved class resembling the fine spaces (for which no uniform characterization is known). The class is closed under forming subspaces.

For each uniform space $\mu X$ there is a coarsest locally fine uniformity $\lambda \mu$ finer than $\mu$. The operator $\lambda$ commutes with completion and preserves both subspaces and uniformly continuous functions. (That is, if $f: \mu X \rightarrow \nu Y$ is uniformly continuous then so is $f: \lambda \mu X \rightarrow \lambda \nu Y$; $\lambda \mu X$ does not have the same uniformly continuous real-valued functions as $\mu X$.) These properties of $\lambda$ are easy to prove once the existence of $\lambda \mu$ is established. The existence proof rests heavily on the lemma, any locally fine uniformity finer than a complete metric uniformity is fine, and on the known results that every uniformity is a union of metric nonseparated uniformities and that every metric space is paracompact. These results are employed also in the generalization of Shirota's theorem. The last section of the paper examines some of the constructions more closely and indicates some more direct proofs.

The first half of the paper is a review of the elementary theory of uniform spaces, supplemented with some results not all of which are related. The best of these results is that every countable uniform covering has a countable uniform star-refinement. As a corollary it follows that a uniformity having a

Received by the editors November 25, 1957 and, in revised form, June 28, 1958.

(1) Research supported (Ginsburg) by University of Miami and the National Cash Register Company, (Isbell) by National Science Foundation through a fellowship and the 1955 Summer Institute, and Office of Naval Research under contracts with George Washington University and Tulane University. 
basis of countable coverings is a union of separable metric nonseparated uniformities. This fact is used in the generalization of Shirota's theorem. Typical of the other results are (i) an explicit description of the equivalence relations corresponding to quotient spaces, and (ii) a proof that Samuel compactification of a complete noncompact space involves adjoining more points than the power of the continuum (generalization of a known result on Stone-Cech compactification).

The authors are indebted to Richard Arens for suggestions which bridged a major gap in our first attempt to generalize Shirota's theorem. (Arens showed us how to construct certain partitions of unity on locally fine spaces. This step is now handled by a construction which works on any uniform space, but Arens' contribution was of critical importance.) We are indebted to Melvin Henriksen and Ernest Michael for contributions which appear below, and the second-named author is indebted also to Pierre Conner, K. Morita, and F. J. Wagner for illuminating discussions of some of these ideas.

1. Coverings. Our terminology and notation for coverings are essentially those of Tukey [17, pp. 43-50]. Generally $u$ denotes a covering of a space $X$; $u=\left\{U_{\alpha} \mid \alpha \in A\right\}$, or $u=\left\{U_{\alpha}\right\}$, for short. We say that $u$ is a refinement of $v$, and write $u<v$, when $u$ and $v$ are coverings of the same set and every element of $u$ is a subset of some element of $v$.

We wish to employ the language of partially ordered sets in connection with coverings ordered by refinement. The language will be more natural if we make use of two simple remarks. First, the relation $<$ (on coverings of a fixed set) is only a quasi-ordering; but there is no difficulty in applying the usual concepts of cofinal set, residual set, filter, to quasi-ordered sets. Second, it is the finer coverings or predecessors under < which are important; in a sense, our quasi-ordered set is ordered downward. Specifically we define a subset $Q$ of a quasi-ordered set $P$ to be cofinal in $P$ if, for every $p$ in $P$, a $q$ in $Q$ can be found satisfying $q \leqq p$. A subset $Q$ of $P$ is residual in $P$ if $q$ in $Q$ and $r \leqq q$ in $P$ imply that $r$ is in $Q . Q$ is a filter in $P$ if (1) $Q$ is an antiresidual subset of $P$, i.e., $q$ in $Q$ and $r \geqq q$ in $P$ imply $r$ is in $Q$, and (2) $Q$ is directed, i.e., for $q$ and $q^{\prime}$ in $Q$, there exists $q^{\prime \prime}$ in $Q$ such that $q^{\prime \prime} \leqq q$ and $q^{\prime \prime} \leqq q^{\prime}$. However, if $P$ is a family of sets ordered by inclusion, one requires that the empty set $\varnothing$ not be an element of $Q$. A filter base is a cofinal subset of a filter.

The following notation is almost identical with that of [17, Chapter V] (which see for definitions). For an intersection $\left\{U_{\alpha} \cap V_{\beta}\right\}$ of coverings, $\left\{U_{\alpha}\right\} \wedge\left\{V_{\beta}\right\}$, or $u \wedge v$. For the star of a set $S$ with respect to a covering $u$, $\operatorname{St}(S, u)$; but in the case of the star of an element $U_{\alpha}$ of $u$ this may be shortened to $U_{\alpha}^{*}$. For the star of a covering $u, u^{*}$. The star-refinement relation $u^{*}<v$ is sometimes written $u<* v$; the convenience of this special symbol will become apparent.

We assume the reader is acquainted with the concepts of star-finite and point-finite [17] and finite order; we also assume an acquaintance with the 
concept of the nerve of a covering [7, pp. 68-70]. Later in the paper we shall be concerned with the uniform structure of the nerve; this will be induced by regarding the nerve as a metric space in which the distance between any two points is the maximum difference in their barycentric coordinates. From now on we shall not speak of order, but use the same terms, "finite-dimensional" or " $n$-dimensional," for a covering as for its nerve.

Besides finiteness we shall need two other concepts involving cardinal numbers, namely countability and nonmeasurability $\left({ }^{2}\right)$.

The following theorem is essentially known.

TheOREM 1.1. Suppose that $u$ and $v$ are coverings of $a$ set and that $v^{*}<u$. If $u$ is (a) finite, (b) star-finite, (c) finite-dimensional, (d) point-finite, or (e) nonmeasurable, then there exists a covering $w$ such that $v<w<* u$ and $w$ has the respective property (a), (b), (c), (d), or (e).

Proof. Let $u=\left\{U_{\alpha}\right\}$ and $v=\left\{V_{\beta}\right\}$. Let $\gamma$ be any subset of $u$ such that there is at least one point common to all members of $\gamma$; and let $C$ be the family of all possible such $\gamma$. For each ordered pair $(\gamma, \delta)$ of elements of $C$, let $W_{\gamma \delta}$ be the union of all $V_{\beta}$ such that

(i) the set of all $U_{\alpha}$ which contain $V_{\beta}$ is precisely $\gamma$, and (ii) the set of all $U_{\alpha}$ which contain $V_{\beta}^{*}$ is precisely $\delta$. Let us call these $V_{\beta}$ parts of $W_{\gamma \delta}$. Let $w=\left\{W_{\gamma^{\delta}}\right\}$.

For any nonempty $V_{\beta}$, the set $\gamma(\beta)$, respectively $\delta(\beta)$, of all $U_{\alpha}$ containing $V_{\beta}$, respectively $V_{\beta}^{*}$, is in $C$. The element $W_{\gamma(\beta) \delta(\beta)}$ of $w$ thus contains $V_{\beta}$, i.e., $v<w$. Given $W_{\gamma \delta}$, let $W_{\sigma \tau} \cap W_{\gamma \delta}$ be nonempty. Then $V_{a} \cap V_{b}$ is nonempty for some part $V_{a}$ of $W_{\sigma \tau}$ and some part $V_{b}$ of $W_{\gamma \delta}$. Any $U_{\alpha}$ in $\delta$ contains $V_{b}^{*}$. Hence $V_{a} \subset U_{\alpha}$. Then $U_{\alpha}$ is in $\sigma$, that is, $\delta$ is a subset of $\sigma$. Therefore $W_{\sigma \tau} \subset U_{\alpha}$. We conclude that $W_{\gamma \delta}^{*} \subset U_{\alpha}$, i.e., $w<* u$.

If $u$ is finite or nonmeasurable, then so is $C$, thus also $w$. Suppose that $u$ is star-finite. Given $W_{\gamma \delta}$, let $U$ in $u$ contain $W_{\gamma \delta}$. Then for every $W_{\lambda \mu}$ meeting $W_{\gamma \delta}$, it is easily seen that both $\lambda$ and $\mu$ are subsets of the star of $U$. Since $u$ is star-finite, the number of $\lambda$ and $\mu$ are finite. Hence only a finite number of $W_{\lambda \mu}$ meet $W_{\gamma \delta}$, i.e., $w$ is star-finite. Finally, if a point $p$ is in at most $n$ members $U_{1}, \cdots, U_{n}$ of $u$, then $p$ is in at most $2^{2 n}$ members of $w$, namely $W_{\lambda \mu}$, where $\lambda$ and $\mu$ are subsets of $\gamma=\left\{U_{i} \mid i \leqq n\right\}$. This proves (c) and (d), and completes the proof.

Tukey proved 1.1 for finite and star-finite coverings [17]. We have not seen the rest of the theorem in the literature.

(2) A cardinal number is nonmeasurable [18] if a set of that power has no ultrafilter (maximal proper filter) of subsets which is closed under countable intersection, other than the trivial ultrafilters which consist of all sets containing a given point; equivalently, there is no nontrivial countably additive two-valued measure, or Ulam measure, on the set. The nonmeasurable numbers are closed under all the operations of transfinite arithmetic. In particular if $m$ is nonmeasurable then so are $2^{m}$ and any $n<m[18]$. It is consistent with the axioms for set theory that no measurable numbers exist [16]. 
It can be shown that 1.1 is not true for countable coverings. However we do have

THEOREM 1.2. Suppose that $u$ and $v$ are coverings of a set such that $v^{* *}<u$. If $u$ is countable, then there exists a countable covering $w$ such that $v<w<* u$.

Proof. Let $u=\left\{U_{i}\right\}$ and $v=\left\{V_{\alpha}\right\}$. Define three functions, $m, n$, and $q$ as follows: $m(\alpha)$ is the least $i$ such that $U_{i}$ contains $V_{\alpha}^{*} ; n(\alpha)$ is the least $i$ such that $U_{i}$ contains $V_{\alpha}^{* *}$; and $q(\alpha)$ is the set of all $i$ such that $U_{i}$ contains $V_{\alpha}$ and $i \leqq n(\alpha)$. Note that $m(\alpha) \leqq n(\alpha)$ and that $m(\alpha)$ and $n(\alpha)$ are elements of $q(\alpha)$. For $j \leqq k$ and $\phi$ a set of integers $\leqq k$ which includes both $j$ and $k$, let $W_{j k \phi}$ be the union of those $V_{\alpha}$ for which $m(\alpha)=j, n(\alpha)=k$, and $q(\alpha)=\phi$. Let us call these $V_{\alpha}$ parts of $W_{j k \phi}$. Clearly $\left\{W_{j k \phi}\right\}=w$ is countable and coarser than $v$. Suppose that the part $V_{a}$ of $W_{j k \phi}$ meets the part $V_{b}$ of $W_{r s \psi}$. Then $V_{a} \subset V_{b}^{*}$, $V_{a}^{*} \subset V_{b}^{* *} \subset U_{s}$, so that $j \leqq s$. Furthermore, $V_{b} \subset V_{a}^{*} \subset U_{j}$. Hence $j$ is in $\psi$. Therefore $W_{\text {rav }}$ is a subset of $U_{j}$, and $W_{j k_{\phi}}^{*} \subset U_{j}$. Consequently $w<* u$.

A covering $u$ is said to be normal with respect to a family of coverings if the family contains an infinite sequence $\left\{u^{i}\right\}$ such that $u^{1}<*$ and for all $n, u^{n+1}<^{*} u^{n}$. From 1.1 we see that if $u$ is normal with respect to a filter of coverings, and $u$ is either finite, star-finite, finite-dimensional, nonmeasurable, or countable, then $u$ is normal with respect to the subfamily consisting of coverings of the same special type as $u$.

2. Basic uniform concepts. The terminology and notations for uniform spaces are taken with slight modifications from Tukey [17].

A preuniformity on a set $S$ is a family $\mu$ of coverings of $S$ which forms a filter in the quasi-ordering $<^{*}$. Specifically $\mu$ is required to satisfy

$\left(U_{\mathbf{i}}\right)$ If $y$ and $z$ are in $\mu$, then $y \wedge z$ is in $\mu$;

$\left(U_{\text {ii }}\right)$ If $y$ is in $\mu$ and $y<z$, then $z$ is in $\mu$;

( $\left.U_{\text {iii }}\right)$ Each element of $\mu$ has a star-refinement in $\mu$.

A uniformity on a topological space is a preuniformity $\mu$ satisfying the two compatibility conditions

( $U_{\text {iv }}$ ) Each element of $\mu$ has a refinement in $\mu$ consisting of open sets; and

$\left(U_{\mathrm{v}}\right)$ For each point $x$ and every open set $U$ containing $x$, some $u$ in $\mu$ can be found so that $\operatorname{St}(x, u) \subset U$.

A preuniformity $\mu$ is said to be a refinement of $\nu$, written $\mu<\nu$, if the family $\mu$ contains the family $\nu$. Every family of (pre)uniformities has a greatest lower bound in the quasi-ordering <; a basis for the greatest lower bound is given by the set of all finite intersections of coverings in the given uniformities. Each family $\left\{\mu_{\alpha}\right\}$ of preuniformities has a least upper bound, namely the family of all coverings normal with respect to the filter $\bigcap_{\alpha} \mu_{\alpha}$. It is not true though that every family of uniformities has a least upper bound uniformity.

A uniformity frequently is said to be separated if the underlying topology is $T_{0}$ (it then follows that the topology is completely regular [2, Chapter IX, p. 10]). Instead, we shall consider only topologies which are $T_{0}$, with a few 
exceptions. These exceptions will be explicitly distinguished as nonseparated. Under this agreement a uniform space $\mu X$ is an ordered pair $(X, \mu)$ where $X$ is a $T_{0}$ space and $\mu$ is a uniformity on $X$; omitting " $T_{0}$ ", one has the definition of a nonseparated uniform space.

This essentially is Tukey's formulation [17]. Bourbaki [2] defines a uniform structure as a filter of reflexive relations in $X$ having a symmetric (filter) basis and such that every relation in the structure extends the square of some relation in the structure. The Tukey coverings are called large coverings; the Bourbaki relations are called entourages. We may pass from the covering $u$ to the relation $R(u)$ of belonging to some common element of $u$; conversely, a relation $R$ on $X$ defines a covering of $X$ consisting of all the left cross sections, i.e., $u(R)=\{R(x) \mid R(x)=\{y \mid x R y\}\}$. Since $u(R(u))$ is a weaker extension than the star, and $R(u(R))$ is a weaker extension than the square, the two formulations are equivalent. We shall use Bourbaki's term, uniform coverings, for Tukey's large coverings.

A suitable preuniformity on a set defines a topology making the whole structure a uniform space. Any preuniformity defines a not necessarily separated uniform space; the stars of a point $X$ with respect to uniform coverings are taken as a basis of neighborhoods of $x$. It is easy to see that this topology will be $T_{0}$ if and only if each $x$ is the intersection of its neighborhoods.

A uniformly continuous function $f: \mu X \rightarrow \nu Y$ is a function $f: X \rightarrow Y$ (into) such that for each covering $V_{\beta}$ in $\nu$, the covering $f^{-1}\left(V_{\beta}\right)$ is in $\mu$. In other words, the inverse image of a uniform covering is uniform. If $f$ is one-to-one onto and $f^{-1}$ is also uniformly continuous, then $f$ is called a uniform equivalence.

The concept of a functor [5] involves certain set-theoretic complications, since it is typically an operator applied to some class larger than a set, such as the class of all uniform spaces. One thinks of a functor as an ordered pair $\left(\lambda, \lambda^{*}\right)$ of functions. However, recalling that a function $f: X \rightarrow Y$ is an ordered triple $(f, X, Y)$, and that ordered pairs and triples of proper classes cannot be formed, we must write the following definition. A functor $h$ on a class $L$ of uniform spaces is a single-valued relation $\lambda \cup \lambda^{*}$ whose domain is the union of the class $L$ and the class $K$ of all uniformly continuous functions whose domains and ranges are elements of $L ; L$ is the domain of $\lambda$ and $K$ is the domain of $\lambda^{*}$. (The partition of $h$ into $\lambda$ and $\lambda^{*}$ can be effected, because the conventions assure that $L$ and $K$ are disjoint.) The values of $\lambda$ are uniform spaces (not necessarily in $L$ ) and the values of $\lambda^{*}$ are uniformly continuous functions. Finally, $h$ must satisfy

(i) for each $f: A \rightarrow B$ in $K$, the domain of $\lambda^{*}(f)$ is $\lambda(A)$ and the range of $\lambda^{*}(f)$ is $\lambda(B)$; and

(ii) for each $f: A \rightarrow B$ and $g: B \rightarrow C$ in $K, \lambda^{*}(g f)=\lambda^{*}(g) \lambda^{*}(f)$.

After defining a functor $\lambda \cup \lambda^{*}$, we may refer to $\lambda$.alone as the functor, $\lambda^{*}$ being understood from the context. Note that the composition of two functors, when the composition is defined, is also a functor. 
When speaking of a subspace of a uniform space we shall always mean a uniform subspace, endowed with the induced topology and the induced uniformity. Usually we shall use the same symbol for the uniformities on the whole space and on the subspace. For a subspace $\mu Y$ of $\mu X$, the uniform coverings of $\mu Y$ are precisely the coverings $\left\{U_{\alpha} \cap Y\right\}$, where $\left\{U_{\alpha}\right\}$ is any uniform covering of $\mu X$.

The Cartesian product $\prod_{\alpha} \mu_{\alpha} S_{\alpha}$ of a family $\left\{\mu_{\alpha} S_{\alpha}\right\}$ of uniform spaces is the product set $\prod_{\alpha} S_{\alpha}$ endowed with the uniformity such that a function into the product is uniformly continuous if and only if each of its coordinate projections is uniformly continuous. A basis for the uniformity may be described as follows. Let $\left\{\mu_{i} S_{i} \mid i \in F\right\}$ be a finite subfamily of the given family. For each $i$ in $F$ let $y^{i}=\left\{Y_{\gamma}^{i}\right\}$ be a uniform covering of $\mu_{i} S_{i}$. The set of all $\prod_{i \in F} Y_{\gamma_{i}} \times \prod_{\alpha \notin F} S_{\alpha}$ for all choices of $Y_{\gamma_{i}} \in\left\{Y_{\gamma}^{i}\right\}, i$ in $F$, is a covering of $\prod_{\alpha} S_{\alpha}$. The family of all such coverings is a basis for $\prod_{\alpha} \mu_{\alpha} S_{\alpha}$. A third way to describe the uniformity for $\prod_{\alpha} \mu_{\alpha} S_{\alpha}$ is to call it the coarsest one making all coordinate projections uniformly continuous.

A quotient $\nu Y$ of $\mu X$ is an identification space of $X$ induced by a uniformly continuous function $f$ on $\mu X$ into $\sigma Z$. The points of $Y$ are the nonempty inverse images $f^{-1}(z)$ of points in $Z$; and the uniformity is the finest one which makes the mapping $k(x)=f^{-1}(f(x))$ of $\mu X$ onto $\nu Y$ uniformly continuous.

The existence of a quotient for each $f$ follows from the existence of greatest lower bounds and the existence of at least one uniformity on $Y$ which makes $k$ uniformly continuous. The existence of the latter is obvious, since $f$ is uniformly continuous.

Conversely, if $\nu Y$ is an identification space of $\mu X$, and the natural mapping $k$ of $\mu X$ onto $\nu Y$ is uniformly continuous, then $\nu Y$ is induced by a mapping $f$ of $\mu X$ into $\sigma Z$ as above, with $f=k$ and $\sigma Z=\nu Y$. This shows that all uniformly continuous functions determine all quotients.

It is natural to ask for explicit conditions on an equivalence relation $R$ on $X$ so that the $R$-equivalence classes actually form a quotient space. We now give such conditions.

Definition. A uniform relation $R$ in a uniform space $\mu X$ is an equivalence relation on $X$ with the property that for each pair of nonequivalent points $x, y$, in $X$, there exists an infinite sequence $\left\{z^{i}\right\}$ of elements of $\mu$ satisfying

(i) if $x^{\prime} R x$ and $y^{\prime} R y$, then $x^{\prime}$ and $y^{\prime}$ are in no common element of $z^{1}$;

(ii) for each element $Z_{0}^{n+1}$ of $z^{n+1}$, an element $Z_{0}^{n}$ of $z^{n}$ can be found such that if the point $p$ is in $Z_{0}^{n+1}$ and $p R q$, then $\operatorname{St}\left(q, z^{n+1}\right) \subset Z_{0}^{n}$.

Definition. The quotient $\mu X / R$ of a uniform space $\mu X$ by a uniform relation $R$ in $\mu X$ is the pair $(X / R, \mu / R)$, where

(a) $X / R$ is the set of equivalence classes of $R$ on $X$;

(b) $\mu / R$ is the set of all coverings of $X / R$ normal with respect to the family of all coverings $\left\{k\left(Z_{\alpha}\right)\right\},\left\{Z_{\alpha}\right\}$ in $\mu, k$ being the natural mapping of $x$ into the equivalence class containing $x$; and 
(c) $X / R$ is endowed with the topology defined by the uniformity $\mu / R$ (see 2.2).

There is no trouble in showing that (regarding a relation as a set of ordered pairs)

2.1. Every intersection of uniform relations is a uniform relation.

THEOREM 2.2. The quotient $\mu X / R$ of a uniform space by a uniform relation $R$ is a uniform space. The natural mapping $k$ of $\mu X$ onto $\mu X / R$ is uniformly continuous. If $f$ is a uniformly continuous function on $\mu X$ into $\nu Y$, then the relation $f(x)=f\left(x^{\prime}\right)$ is a uniform relation $R$, and the function $f^{*}$ on $\mu X / R$ into $\nu Y$, defined by $f k^{-1}$, is uniformly continuous.

Proof. To prove that $\mu X / R$ is a uniform space, it is sufficient to show that $\mu / R$ is nonempty and makes $X / R$ a $T_{0}$ space. By (ii) of the definition of a uniform relation, $\left\{k\left(z^{n}\right)\right\}$ is an infinite sequence of successive star-refinements. Hence $k\left(z^{1}\right)$ is in $\mu / R$. By (i), if $k(x) \neq k(y)$, then $k(y)$ is not in $\operatorname{St}\left(k(x), k\left(z^{1}\right)\right)$. Hence any pair of points in $X / R$ are separated, i.e., $X / R$ is $T_{0}$.

It is obvious that $k$ is uniformly continuous.

Let $f$ be uniformly continuous and $x^{\prime} R x$ the relation $f\left(x^{\prime}\right)=f(x)$. Suppose that $f(x) \neq f(y)$. Choose a sequence $\left\{v^{n}\right\}$ in $\nu$ such that $f(y)$ is not in $\operatorname{St}\left(f(x), v^{1}\right)$ and $v^{n+1}<v^{*}$ for each $n$. Then, as is easily seen, $z^{n}=f^{-1}\left(v^{n}\right)$ satisfies (i) and (ii). It follows that the quotient is the one constructed previously and $f^{*}$ is uniformly continuous (this could be proved directly). Hence the theorem.

At this point we wish to acknowledge a contribution of Ernest Michael. Michael pointed out to us the first definition of quotients given above, in terms of "the finest uniformity such that ..." ; we had previously worked out the approach of 2.2. The two approaches are clearly equivalent.

A Cauchy filter in $\mu X$ is a filter of subsets of $X$ which contains an element of every covering in $\mu$. A filter converges to $x$ if it contains as an element each neighborhood of $x$. A complete space is one in which every Cauchy filter converges.

A space is uniformly locally compact if it has a uniform covering consisting of compact sets; and similarly for other "uniform local" properties. A collection $\left\{S_{\alpha}\right\}$ of subsets of a uniform space $\mu X$ is uniformly discrete if there exists a uniform covering $u$ such that the sets $\operatorname{St}\left(S_{\alpha}, u\right)$ are pairwise disjoint. A subspace is called uniformly discrete if its points form a uniformly discrete family. A countable union of uniformly discrete sets is said to be $\sigma$-uniformly discrete.

Let $\left\{\mu_{\alpha} S_{\alpha}\right\}$ be a family of uniform spaces. For each $\alpha$ let $\nu_{\alpha} T_{\alpha}$ be a uniform equivalent of $\mu_{\alpha} S_{\alpha}$ such that $T_{\alpha_{1}} \cap T_{\alpha_{2}}$ is empty for $\alpha_{1} \neq \alpha_{2}$. The $\operatorname{sum} \sum_{\alpha} \mu_{\alpha} S_{\alpha}$ is the union of the $T_{\alpha}$ endowed with the following uniformity. A covering of $\sum \mu_{\alpha} S_{\alpha}$ is uniform if and only if its restriction to each summand $T_{\alpha}$ is uniform in $\nu_{\alpha}$.

Example A. For each countable ordinal $\alpha$ let $X_{\alpha}=\{(\xi, \alpha) \mid \xi$ is an ordinal 
$\leqq \alpha\}$. Let $(\xi, \alpha) \leqq(\gamma, \alpha)$ if and only if $\xi \leqq \gamma$. In the order topology, $X_{\alpha}$ is a compact space and thus has a unique uniformity $\mu_{\alpha}$ consisting of all open coverings.

Let $\mu X$ be the sum of the family $\left\{\mu_{\alpha} X_{\alpha} \mid \alpha<\omega_{1}\right\}$. Let $R$ be the equivalence relation defined by $(\xi, \alpha) R(\eta, \beta)$ if $\xi=\eta$. Let $Y$ be the set of all countable ordinal numbers with the order topology. $Y$ has a unique uniformity, with a basis consisting of all finite open coverings of $Y$. Let $f$ be the function from $\mu X$ onto $\nu Y$ defined by $f(\xi, \alpha)=\xi$. It is easily seen that $f$ is uniformly continuous and induces the relation $R$. Thus $R$ is a uniform relation. Note that $\mu X / R$ is uniformly equivalent to $\nu Y$.

The primary point we wish to note is that under the natural mapping $k$ of $\mu X$ onto $\mu X / R$, the image of a uniform covering is not necessarily uniform. This is shown by the covering $\left\{X_{\alpha}\right\}$. Clearly $\left\{k\left(X_{\alpha}\right)\right\}$ has no finite (or countable) subcovering. Another point we wish to note is that $\mu X$ is complete but $\mu X / R$ is not. It is known [2] that this can happen in a topological linear space; here we see it happening in a uniformly locally compact space. In passing we note that $\mu X / R$ is a direct limit of the compact spaces $\mu_{\alpha} X_{\alpha}$.

2.3. For any uniform space $\mu X$ and any cardinal number $m$, either $\mu X$ has a uniformly discrete subspace of power $m$ or every uniform covering has a uniform refinement of power $<m$.

Proof. Let $u$ be any uniform covering and $v^{* * *}<u, v$ in $\mu$. Using transfinite induction, construct in the obvious way a maximal set of points $E=\left\{p_{\gamma}\right\}$ such that the sets $\operatorname{St}\left(p_{\gamma}, v\right)$ are pairwise disjoint. Clearly $E$ is uniformly discrete. Let $w=\left\{\operatorname{St}\left(p_{\gamma}, v^{* * *}\right)\right\}$. Let $x$ be any point of $X$. Due to the maximality of $E, \operatorname{St}(x, v) \cap \operatorname{St}\left(p_{\gamma}, v\right)$ is nonempty for some $p_{\gamma}$ in $E$. For this $p_{\gamma}, x$ is in $\operatorname{St}\left(p_{\gamma}, v^{* * *}\right)$. Thus $w$ is a covering; by a similar argument $v<w<u$, and $w$ has the same power as $E$. In particular, either $E$ has power $\geqq m$ or $w$ has power $<m$.

3. Some basic operators. This section discusses six functors, together with some remarks on successive application of these functors. We first consider the completion as a functor.

We shall not construct the completion of a uniform space since that is done in [2] and in [17]. In both places, equivalence classes of Cauchy filters are used. Two Cauchy filters, $D$ and $E$, are equivalent if the filter $D \vee E$, consisting of all $A \cup B$, where $A$ is in $D$ and $B$ in $E$, is Cauchy. Note that each equivalence class of Cauchy filters contains a unique minimal element (minimal with respect to set inclusion). Bourbaki [2, Chapter IX, pp. 14-15] points this out in a special case. A filter $D$ is said to be uniform in $\mu X$ if for each $A$ in $D$ there exist $B$ in $D$ and $u$ in $\mu$ such that $\operatorname{St}(B, u) \subset A$. Note that each equivalence class of Cauchy filters contains exactly one uniform filter. This is the family of all $\operatorname{St}(B, u), u$ in $\mu, B$ in $D$, for any representative $D$ of the equivalence class.

We designate the completion of a uniform space $\mu X$ as $\pi \mu X$. It is well 
known that each uniformly continuous function $f$ on $\mu X$ into $\nu Y$ has a unique extension $\vec{f}$ over $\pi \mu X$ into $\pi \nu Y$ [2, Chapter II, p. 101]. The completion $\pi$, together with the single-valued relation taking each $f$ to $\bar{f}$, constitutes a functor on the class of all uniform spaces.

Since the completion of a subspace of $\mu X$ is the closure of the subspace in $\pi \mu X, \pi$ preserves subspaces. In view of Example $\mathrm{A}, \pi$ does not preserve quotients.

One fundamental property of the completion which will be needed is Morita's Lemma :

Lemma (MoRita). For any uniform covering $\left\{U_{\alpha}\right\}$ of $\mu X$, let $U_{\alpha}^{\prime}$ (for each $\alpha)$ consist of $U_{\alpha}$ together with all points of $\pi \mu X$ which are not limit points of $X-U_{\alpha}$; then $\left\{U_{\alpha}^{\prime}\right\}$ is a uniform covering of $\pi \mu X$, and the family of all such coverings is a basis for $\pi \mu$.

The proof is in [10].

Other important functors are obtained by replacing $(X, \mu)$ with $(X, h \mu)$, where $h \mu$ is a suitable subfamily of $\mu$. Tukey showed $[17$, pp. 44-49; 57-58] that the finite uniform coverings of any uniform space $\mu X$ form a basis for a uniformity $f \mu$. In view of 1.1 and 1.2 , the nonmeasurable and the countable uniform coverings form bases for preuniformities $n \mu, e \mu$; since each of these contains $f \mu$, they are uniformities. Similarly those uniform coverings which are at once countable and star-finite form a basis for a uniformity, which we shall call $s \mu$. The mappings $n, e, f, s$, which take $\mu$ into $n \mu, e \mu, f \mu$, and $s \mu$ respectively, are functors. (The mapping $\lambda^{*}$ in each case is the identity. The conditions on $\lambda^{*}$ are satisfied since, clearly, if $f$ is uniformly continuous from $\mu X$ into $\nu Y$, then $f$ is uniformly continuous from $h \mu X$ into $h \nu Y, h$ being $n, e, f$, or $s$.

A uniformity is precompact if it is coarser than $f$, i.e., if every uniform covering has a uniform finite subcovering. This is equivalent to each of the following conditions: (i) the completion is compact; and (ii) each ultrafilter (=maximal proper filter) is Cauchy.

A metric uniformity is one which has a countable basis. A metric space $(d, X)$ induces a uniformity with a basis $\left\{u^{n}\right\}$, where $u^{n}$ is the covering by open spheres of radius $1 / n$. When we speak of a metric space we mean a metric uniform space. It is a known fact [2, Chapter IX, p. 23] that a metric uniformity is induced by a metric. Unless specified otherwise, when we speak of the real line $R$, we mean $R$ with the usual metric uniformity, $m$. In a metric uniformity precompactness is equivalent to being totally bounded. For proofs and further facts see [2] and [17].

We now quote Weil's uniform version of Urysohn's Lemma [2, Chapter IX, p. 9].

Theorem (WeIL). For every subset $A$ of a uniform space $\mu X$ and every uniform covering $u$, there is a uniformly continuous real-valued function $f$ on 
$\mu X$ such that $f(x)=1$ for each $x$ in $A$ and $f(x)=0$ for each $x$ in $X-\operatorname{St}(A, u)$.

Given a uniform space $\mu X$, let $c \mu$ be the filter having a basis consisting of all finite intersections of the inverse images, under uniformly continuous real-valued functions, of uniform coverings in $m R$. It is obvious that $c \mu$ satisfies the conditions $\left(U_{\mathrm{i}}\right),\left(U_{\mathrm{ii}}\right)$, and $\left(U_{\mathrm{iv}}\right)$ for a uniformity; $\left(U_{\mathrm{v}}\right)$ is a consequence of Weil's Theorem, and $\left(U_{\mathrm{iii}}\right)$ may be inferred from the observation that the basis described above can also be described in terms of uniformly continuous mappings into Euclidean spaces (finite products of lines). Thus $c \mu$ is a uniformity. Clearly the single-valued relation $c$ which associates to each space $\mu X$ the space $c \mu X$, and to each $f: \mu X \rightarrow \nu Y$ the mapping $f: c \mu X$ $\rightarrow c \nu Y$, is a functor.

The preceding paragraph suffices to define and establish the existence of the functor $c$. The question arises, what are reasonable sufficient conditions for a uniform covering of $\mu X$ to be uniform on $c \mu X$ ? No generally satisfactory conditions are known. The following result will be enough for the purposes of this paper. A uniform covering of $\mu X$ whose nerve is uniformly equivalent to a subspace of some Euclidean space is a uniform covering of $c \mu X$. This is part of 1.6 of [8], and will be cited again in the proof of Theorem 4.10 below.

We already have fairly explicit descriptions for the functors previously introduced. We add a detail concerning the effect of $e$. The following result is well known, except for the bracketed additions.

3.1. For every [every countable] uniform covering $u$ of a uniform space $\mu X$, there is a uniformly continuous mapping of $\mu X$ into a [separable] metric space such that $u$ is refined by the inverse image of some uniform covering of the range.

The proof for general $u$ (see e.g. [8]) depends on a normal sequence $\left(u^{n}\right)$ beginning with $u$. If $u$ is countable, then by 1.2 we may take every $u^{n}$ to be countable. In this case it is clear from the construction that the range contains no uncountable set of points with mutual distances bounded away from zero, and it is well known that a metric space having this property is separable.

Now consider the functors $n, e, f, s, c$, and $\pi$. The first three and the last preserve subspaces. We shall see that $s$ and $c$ do not preserve subspaces. The first five functors preserve the points of a space, thus preserving images. The first five functors also preserve quotients, as is easily worked out. In view of Example $A, \pi$ preserves neither images nor quotients.

The first five functors coarsen the structure. Thus they must preserve Cauchy filters, and cannot create new uniform filters. We shall be interested in the question to what extent these functors create new Cauchy filters. Note that $f$ does create many new Cauchy filters. In fact, every ultrafilter in $f \mu X$ is Cauchy. If this condition is also true in $\mu X$, then $\mu X$ is precompact, i.e., $f \mu=\mu$.

The composite functor $\pi f$ is called the Samuel compactification [13].

We shall abbreviate $\pi f$ by $\beta$, i.e., $\pi f \mu X=\beta \mu X$. This is in analogy with the 
standard notation $\beta X$ for the Stone-Čech compactification. The Stone-Øech compactification of a completely regular space is characterized as a compact space containing the given space as a dense subspace and admitting an extension of all continuous, bounded, real-valued functions. The Samuel compactification may be similarly characterized as a compact space $Y$ (with its unique uniformity) for which the following statement is true. There is a one to one uniformly continuous function $i$ mapping $\mu X$ upon a dense subspace of $Y$ such that every bounded uniformly continuous real-valued function on $\mu X$ may be extended continuously over $Y . \beta X$, of course, is $\beta a X$, where $a$ is the finest uniformity compatible with the topology of $X$.

Clearly $\mu<n \mu<e \mu<s \mu<c \mu<f \mu$, for each $\mu$. Furthermore, $n \mu, e \mu$, and $f \mu$ are the l.u.b.'s of $\mu$ and, respectively, $n a, e a, f a$.

To see this consider, for example, $e \mu$. It is coarser than both $e a$ and $\mu$, that is, it is a subset of the intersection of these families of coverings. Let $\nu$ be any uniformity coarser than both $e a$ and $\mu$. We shall show that every covering in $\nu$ has a countable refinement in $\mu$. By 2.3 , either every covering in $\nu$ has a countable refinement in $\nu$ (a subset of $\mu$ ) whence we are through, or else $\nu X$ contains an uncountable uniformly discrete subspace $Y$. If the latter is true, then since $e a<\nu, Y$ is uniformly discrete in $e a X$, which is impossible.

A similar cardinality argument applies for $f \mu$ and $n \mu$.

The corresponding statement for $s \mu$ and $c \mu$ is false, as the next example shows.

EXAMPLE B. Let $X$ be the set of all sequences $x=\left\{x_{n}\right\}$ of real numbers such that (i) $0 \leqq x_{n} \leqq 1$ for all $n$, and (ii) $x_{n}=0$ for all $n$ except possibly one value. Under the distance function $d(x, y)=\sum_{n}\left|x_{n}-y_{n}\right|$, where $x=\left\{x_{n}\right\}$ and $y=\left\{y_{n}\right\}, X$ is a metric space $\mu X$.

Let $u=\left\{U_{\alpha}\right\}$ be any star-finite uniform covering. There exists $\epsilon>0$ such that the family of $\epsilon$-spheres refines $\left\{U_{\alpha}\right\}$. Suppose that $U_{0}$ is a $U_{\alpha}$ which contains the $\epsilon$-sphere about 0 , the sequence of all zeros. One easily verifies that every $U_{\alpha}$ is joined to $U_{0}$ by a chain of intersecting sets $U_{\beta}$ of length at most $[1 / \epsilon]+1$. Since $u$ is star-finite, it follows that $u$ is finite. Hence s $\mu$ $=c \mu=f \mu$.

Now let $\left\{U_{\alpha}\right\}$ be any open covering of $X$. One of the $U_{\alpha}$ contains a $\delta$-sphere about 0 , for some $\delta>0$. Let $V$ be the $\delta$-sphere about 0 . For each $k$ let $A_{k}=\left\{x \mid \delta / 2<x_{k} \leqq 1\right\}$. Then $A_{k}$ is an open subset of $X$ and $A_{k} \cap A_{j}$ is empty for $j \neq k$. There exists a denumerable covering $v^{k}=\left\{V_{n}^{k}\right\}$ of $A_{k}$ such that

(1) each $V_{n}^{k}$ is a subset of some $U_{\alpha}$,

(2) no three elements of $v^{k}$ have a common point, and

(3) each $V_{n}^{k}$ is a subset of $A_{k}$.

(The construction of $v^{k}$ is easy since the closure of $A_{k}$ is a line segment.) Clearly $w=\{V\} \cup\left(\bigcup_{k} v^{k}\right)$ is a covering of $X$ which refines $\left\{U_{\alpha}\right\}$. Furthermore $w$ is countable, star-finite, and one-dimensional. Thus $c a<\mu$ and $s a<\mu$. Hence $\mu$ is the least upper bound of $c a$ and $\mu$ ( $s a$ and $\mu$ ). 
It is easily seen that $c \mu=s \mu=f \mu \neq \mu$.

We now shall see that $s$ and $c$ fail to preserve subspaces in Example B. To this end let $Y$ be the subspace consisting of the points $x^{n}=\left\{x_{i}^{n}\right\}$, where each $x_{n}^{n}=1$. As a subspace of $\mu X, Y$ is an infinite, uniformly discrete subset. Furthermore, $\mu Y=a Y$ and $s a Y=c a Y=a Y$. From this it follows that sa $Y$ $=c a Y$ is not precompact. On the other hand, since $s \mu X$ and $c \mu X$ are $f \mu X$, i.e., are precompact, so are all their subspaces, i.e., so are $(s \mu X) \cap Y$ and $(c \mu X) \cap Y$.

Example B also shows that $c$ and $\pi$ do not commute. Clearly $\mu X$ is complete. Thus $c \pi \mu X=c \mu X=f \mu X$. On the other hand, $\pi c \mu X=\pi f \mu X=\beta \mu X$ is compact, which $f \mu X$ is not. The same situation prevails with any complete noncompact bounded convex $\left(^{3}\right)$ (hence infinite-dimensional) metric space. In this connection we mention an unsolved problem raised by Henriksen [1, p. 122]: Is the completion of $\mu X$ always homeomorphic with the completion of the least upper bound of $c a$ and $\mu$ ? An affirmative answer would imply that the functors $\pi$ and $e$ commute. This also is an unsolved problem.

The completions of $\mu X, n \mu X, e \mu X, s \mu X, c \mu X$ and $f \mu X$ are topologically subspaces of each other in the order given, all being contained in the last, $\pi f \mu X$. The reason is that all of these functors $(n, e, s, c, f)$ preserve uniform filters. Since $f$ is the strongest, it suffices to verify this for $f$. Let $D$ be a uniform filter in $\mu X$, and let $A$ be any element of $D$. There exist $B$ in $D$ and $u$ in $\mu$ such that $\operatorname{St}(B, u) \subset A$. Let $v$ be the covering in $f \mu$ consisting of the two elements (i) $\operatorname{St}(B, u)$, and (ii) the union of the remaining elements of $u$. Clearly $\operatorname{St}(B, v)=\operatorname{St}(B, u)$ and $u<v$. Thus $\operatorname{St}(B, v) \subset A$, i.e., $D$ is uniform in $f \mu$.

A similar argument shows that a uniform relation in $\mu X$ is uniform in $f \mu X$. Also, it is easy to deduce that a uniform relation in $\mu X$ may be extended over $\pi \mu X$, and hence over $\beta \mu X$. We elaborate on the details.

TheOREM 3.2. A uniform relation $R$ in a uniform space $\mu X$ can be extended to a uniform relation on $\beta \mu X$. If $R^{*}$ denotes the intersection of all such extensions of $R$, then $\beta \mu X / R^{*}=\beta(\mu X / R)$.

Proof. Let $k$ be the uniformly continuous function which maps $\mu X$ onto $\mu X / R=\nu Y$. Clearly $k$ is also uniformly continuous from $f \mu X$ onto $f \nu Y$, and thus from $f \mu X$ into the complete space $\beta \nu Y$. Since $X$ is dense in $\beta \mu X, k$ has a unique uniformly continuous extension, call it $j$, on $\beta \mu X$ into $\beta \nu Y$. Since $j(\beta \mu X)$ is both compact and dense in $\beta \nu Y$, the function $j$ is onto $\beta \nu Y$. Let $R^{*}$ be the uniform relation on $\beta \mu X$ determined by $j$ and let $f$ be the natural mapping of $\beta \mu X$ onto $\beta \mu X / R^{*}$. Obviously $j f^{-1}$ is a one to one uniformly continuous function of $\beta \mu X / R^{*}$ onto $\beta(\mu X / R)$. Since $\beta \mu X / R^{*}$ is compact and $\beta(\mu X / R)$ is a Hausdorff space, $j f^{-1}$ is a homeomorphism, and thus an open

(3) A metric space is called convex if every two points are contained in a subspace isometric with a line segment. 
mapping. From this it readily follows that $\left(j f^{-1}\right)^{-1}$ is also uniformly continuous, i.e., $\beta \mu X / R^{*}=\beta(\mu X / R)$.

Now let $R^{\prime}$ be any other uniform relation which extends $R$ over $\beta \mu X$. Then $R^{\prime}$ defines a quotient space $Z$ and a uniformly continuous function $k^{\prime}$ of $\beta \mu X$ onto $Z$. Hence $Z$ is compact. The restriction of $k^{\prime}$ to $X$ is a function $i$ which is uniformly continuous on $f \mu X$, thus uniformly continuous on $\mu X$. Since $R^{\prime}$ extends $R$, the quotient of $\mu X$ determined by $i$ is $\nu Y$. Then $i=i^{*} k$, where $k$ is the quotient mapping deduced from $R$ and $i^{*}$ is some uniformly continuous function on $\nu Y$ into $Z$. $Z$ being compact, each covering of $Z$ is refinable by a finite covering. Hence $i^{*}$ is uniformly continuous on $f \nu Y$ and thus extends over $\beta \nu Y$. Let $i^{\prime}$ be the uniformly continuous extension of $i^{*}$ over $\beta \nu Y$. Then $k^{\prime}$ and $i^{\prime} j$ are continuous functions which coincide on the dense subset $\mu X$ of $\beta \mu X$. Hence $k^{\prime}$ and $i^{\prime} j$ are identical. This means that $R^{\prime}$ is a stronger equivalence relation than $R^{*}$, as was to be shown.

Constructing the Samuel compactification of a complete noncompact space (as with the Stone-Cech compactification [3]) involves adjoining a huge number of points, none of which can satisfy the first axiom of countability. This is seen by applying 2.3 , a theorem of Pospišil [6], and a theorem of Katètov [9] as follows. Let $\mu X$ be complete and noncompact. Then $\mu X$ is not precompact, and by 2.3 , it contains a denumerably infinite uniformly discrete subspace $a N$. Every real-valued function on the set $N$ is, of course, uniformly continuous. According to Katětov's theorem every bounded uniformly continuous function on a subspace of a uniform space has a bounded uniformly continuous extension over the entire space. Hence every bounded function on $N$ has a continuous extension over $\beta \mu X$. Hence the closure of $N$ in $\beta \mu X$ is a compact extension of $N$ over which every bounded continuous function on $N$ extends, i.e., it is the Stone-Cech compactification $\beta N$. Pospišil showed that the number of points of $\beta N$ is $2^{2 N_{0}}$ [6]. Here $a N$ is complete and therefore closed in $\mu X$. Thus all but countably many points of $\beta N$, i.e., except $N$ itself, are in $\beta \mu X-X$. To see that the first axiom of countability cannot hold at any point of $\beta \mu X-X$, it suffices to show that every closed $G_{\delta}$ set of $\beta \mu X$, which is disjoint from $X$, is infinite. (This suffices since a point satisfying first countability is a closed $G_{\delta}$.) Now observe that any closed $G_{\delta}$ set $H$ of the compact space $\beta \mu X$ is the set of zeros of some continuous real-valued function $f$. Let $\left\{x_{n}\right\}$ be a sequence of points of $X$ such that $f\left(x_{n}\right)$ converges to $0 .\left\{x_{n}\right\}$ exists since $X$ is dense in $\beta \mu X$ and $f$ is continuous. All the limit points of $\left\{x_{n}\right\}$ are contained in the zeros of $f$, i.e., in $H$, which is in $\beta \mu X-X$. Thus the subspace of $\mu X$ consisting of all $x_{n}$ is closed, and hence complete in $\mu X$. Clearly it is not compact. Hence by 2.3 , it has an infinite uniformly discrete subspace $M=\left\{x_{n_{i}}\right\}$. As above we obtain the topological space $\beta M(=\beta N)$. Here $\beta M-M$ is in $H$. Summarizing we have

Theorem 3.3. If $\mu X$ is complete but not compact, then $\beta \mu X-X$ contains at least $2^{2 N_{0}}$ points. No point in $\beta \mu X-X$ satisfies the first countability axiom. 
Any closed $G_{\delta}$ of $\beta \mu X$ which is disjoint from $X$ contains the topological space $\beta N-N$.

4. Locally fine spaces. A uniform space $\mu X$ is locally fine provided every uniformly locally uniform covering is uniform. A covering $\left\{W_{\gamma}\right\}$ is uniformly locally uniform in case there exists a uniform covering $\left\{U_{\alpha}\right\}$ such that on each of the subspaces $U_{\alpha}$ the covering $\left\{U_{\alpha} \cap W_{\gamma}\right\}$ is uniform (in the induced uniformity). This means that for each fixed $\alpha$ we may replace $\left\{W_{\gamma}\right\}$ with a uniform covering $w^{\alpha}=\left\{W_{\beta}^{\alpha}\right\}$ so that $\left\{U_{\alpha} \cap W_{\beta}^{\alpha}\right\}=\left\{U_{\alpha} \cap W_{\gamma}\right\}$ ( $\beta$ and $\gamma$ vary here). Evidently $\mu X$ is locally fine if and only if it is closed under the "staggered intersection" operation $\left\{U_{\alpha} \cap W_{\beta}^{\alpha} \mid\right.$ all $\alpha$, all $\left.\beta\right\}$.

We shall next construct a locally fine space $\lambda \mu X$ which is naturally associated with an arbitrary space $\mu X$. In fact the construction is a direct transfinite iteration of the operation just described. However, our proof that $\lambda \mu$ is a uniformity involves an auxiliary transfinite induction; and to simplify the argument we shall treat metric spaces before the general case. One accompanying complication is that we shall have to handle various filters of coverings, some of which will finally turn out to be uniformities.

Accordingly we begin by defining, for any filter of coverings $\mu$, the derivative $\mu^{(1)}$ of $\mu$ as the family of all coverings which have refinements of the form $\left\{U_{\alpha} \cap W_{\beta}^{\alpha}\right\}$, where $\left\{U_{\alpha}\right\}$ is in $\mu$ and for each fixed $\alpha$ the family $\left\{W_{\beta}^{\alpha}\right\}$ is in $\mu$. Evidently $\mu^{(1)}$ is again a filter of coverings. Thus we may define $\mu^{(\alpha)}$ for each ordinal number $\alpha$, by the inductive rules $\mu^{(\alpha+1)}=\left(\mu^{(\alpha)}\right)^{(1)}$ and for limit ordinals $\beta, \mu^{(\beta)}$ is the union of all previous $\mu^{(\alpha)}$. Since the successive derivatives $\mu^{(\alpha)}$ are expanding, evidently there is a last derivative $\mu^{(\alpha)}=\mu^{(\alpha+1)}$. The last derivative of $\mu$ is written $\lambda \mu$.

We have no counterexample to the conjecture that when $\mu$ is a uniformity, so is $\mu^{(1)}$, and hence all $\left.\mu^{(\alpha)}{ }^{4}\right)$. We shall return to this question later in the paper.

4.1. Lemma. Let $\mu X$ be a uniform space. Let $P$ be a partially ordered set and $h$ a mapping of $P$ into $2^{X}$ (the set of subsets of $X$ ). Let $S$ be a subset of $P$ such that every chain in $P-S$ is finite. Suppose that (1) the set of all $h(p), p a$ maximal element of $P$, is a uniform covering of $\mu X$, and (2) for each $p$ in $P$ the set of all $h(q) \cap h(p), q$ an immediate successor of $p$, is a uniform covering of $h(p)$ (in the induced uniformity). Then the set of all $h(s), s$ in $S$, is a covering of $X$ and an element of $\lambda \mu$.

Proof. The lemma is certainly true if every chain in $P-S$ is of length at most 1 ; then $\{h(s) \mid s \in S\}$, is in $\mu^{(1)}$. (It is uniform on each $h(p)$, $p$ maximal in $P$; for either $p$ itself is in $S$ or all its successors are in $S$.) For a proof by induction, let us define an ordinal-valued function $\delta$ on $P-S$ as follows. If every successor of $P$ is in $S$, then $\delta(p)=0$. If $\delta(q)$ is defined for every successor

(4) In a preliminary announcement $[1$, p. 106] we erroneously claimed a proof of this. 
$q$ of $p$ which is not in $S$, then $\delta(p)$ is the least ordinal greater than all these $\delta(q)$. This defines $\delta$ on all of $P-S$; otherwise $\delta$ would be undefined on some $p_{1}$, on a successor $p_{2}$, and so on through an infinite chain in $P-S$, which is impossible. Now by induction on $\delta(p)$, the family $\{h(s) \cap h(p) \mid s \in S\}$ is a covering in the last derivative of the induced uniformity on $h(p)$; for (a) it is coarser than $\{(h(q) \cap h(p)) \cap h(s) \mid q<p, s \in S\}$; (b) the family $\{h(q) \cap h(p)\}$ is uniform on $h(p)$ by hypothesis (2), and (c) for each $q,\{h(s) \cap h(q)\}$ is in the last derivative of the induced uniformity on $h(q)$ by induction. Thereforc by hypothesis (1), $\{h(s)\}$ is in $\lambda \mu$, as required.

\subsection{For any complete metric space $\mu X, \lambda \mu=a$.}

Proof. It is well known that for a metrizable space $X, a$ consists of all coverings which have open refinements. Every covering in $\mu$ has an open refinement. This property is evidently preserved in taking derivatives, and therefore $\lambda \mu$ is contained in $a$.

For the converse, let $\left\{V_{\beta}\right\}$ be any open covering of $X$. For each $n$, let $u^{n}=\left\{U_{n, \alpha}\right\}$ be a uniform covering of $\mu X$ consisting of sets of diameter at most $2^{-n}$. Partially order the set $P$ of all indices $(n, \alpha)$ as follows: $(n+1, \beta)$ $<(n, \alpha)$ if $U_{n+1, \beta}$ meets $U_{n, \alpha} ;(n+k, \gamma)<(n, \alpha)$ if there is a chain $(n+k, \gamma)$ $<(n+k-1, \delta)<\cdots<(n, \alpha)$. Let $S$ be the set of all $(n, \alpha)$ such that $\operatorname{St}\left(U_{n, \alpha}, u^{n}\right)$ is a subset of some $V_{\beta}$. Since $\mu X$ is complete and each $U_{n, \alpha}$ is of diameter at most $2^{-n}$, each infinite descending chain in $P$ converges to a limit point $x$. Since some $\epsilon$-neighborhood of $x$ is in some $V_{\beta}$, only a finite number of the $U_{n, \alpha}$ in the chain can be in $P-S$. The remaining hypotheses of 4.1 are obvious. Therefore $y=\left\{U_{n, \alpha} \mid(n, \alpha) \in S\right\}$ is in $\lambda \mu$. Since $y<\left\{V_{\beta}\right\},\left\{V_{\beta}\right\}$ is in $\lambda \mu$.

4.3. For any uniform space $\mu X$, every covering in $\mu$ is normal with respect to the family of all point-finite coverings in $\lambda \mu$.

Proof. By 3.1, for each covering $u$ in $\mu$, a uniformly continuous mapping $f$ of $\mu X$ into a metric space $\nu Y$ and a uniform covering $v$ of $\nu Y$ can be found such that $f^{-1}(v)$ is a refinement of $u$. We may take $\nu Y$ to be complete. Since every metrizable space is paracompact [15], there is a normal sequence of locally finite open coverings of $Y,\left(v^{n}\right)$, with $v^{1}=v$. Each $v^{n}$ is in $\lambda \nu$, by 4.2. By an evident induction, if $v^{n}$ is in $\nu^{(\alpha)}$, then $f^{-1}\left(v^{n}\right)$ is in $\mu^{(\alpha)}$. Evidently $f^{-1}\left(v^{n}\right)$ is point-finite, and $\left(f^{-1}\left(v^{n}\right)\right)$ is a normal sequence, as required.

REMARK. The coverings just constructed are locally finite. We wish to emphasize the property of point-finiteness, so that we can use 1.1 in the next proof.

Theorem 4.4. For every uniform space $\mu X, \lambda \mu$ is a uniformity; it is the coarsest locally fine uniformity finer than $\mu$. Every locally fine uniformity has a basis of uniformly locally finite $\sigma$-uniformly discrete coverings. $\lambda$ is a functor preserving subspaces and commuting with completion.

Proof. Let us abbreviate "normal with respect to $\lambda \mu$ " by "normal." We 
prove by induction that every covering in $\lambda \mu$ has a point-finite normal refinement. By 4.3 this is true for coverings in $\mu$. Let $\alpha$ be the least ordinal such that the assertion fails for some $u$ in $\mu^{(\alpha)}$. We may suppose $u$ has the form $\left\{V_{\beta} \cap W_{\gamma}^{\beta}\right\}$, where $\left\{V_{\beta}\right\}$ and each $w^{\beta}=\left\{W_{\gamma}^{\beta}\right\}$ are in previous derivatives of $\mu$. Thus $\left\{V_{\beta}\right\}$ has a point-finite refinement $\left\{A_{\delta}\right\}$ which has a pointfinite normal star-refinement $\left\{Y_{\rho}\right\}$. For each $\delta$ choose one $\beta$ such that $V_{\beta}$ contains $A_{\delta}$. Since $\left\{A_{\delta}\right\}$ is point-finite, each $Y_{\rho}$ is contained in only finitely many $A_{\delta}$; let $b^{p}$ be the intersection of the corresponding coverings $w^{\beta}$. Since each $\mu^{(\alpha)}$ is a filter, the inductive hypothesis implies that $b^{p}$ has a pointfinite normal star-refinement $\left\{Z_{\sigma}^{\rho}\right\}$. Computation shows that $\left\{Y_{\rho} \cap Z_{\sigma}^{\rho}\right\}$ is a point-finite star-refinement of $u$. Moreover, since $\left\{Y_{\rho}\right\}$ and each $\left\{Z_{\sigma}^{\rho}\right\}$ are normal, the construction may be continued, demonstrating that $\left\{Y_{\rho} \cap Z_{\sigma}^{\rho}\right\}$ is normal.

Thus $\lambda \mu$ is a uniformity. By construction it is locally fine and finer than $\mu$. Moreover, any locally fine uniformity finer than $\mu$ is finer than $\mu^{(1)}$ and, by induction, finer than $\lambda \mu$.

In particular, if $\mu$ is locally fine then $\mu=\lambda \mu$. Then every uniform covering has a uniform refinement which is uniformly locally finite and $\sigma$-uniformly discrete because of $3.1,4.2$, and the known fact that this is true in spaces $a X$ when $X$ is fully normal [15].

Obviously $\lambda$ is a functor and preserves subspaces. Since $\lambda \pi \mu X$ is $\pi \mu X$ with a finer uniformity, it is complete; and since $\lambda$ preserves subspaces and topology, $\lambda \pi \mu X$ is a complete space containing $\lambda \mu X$ as a dense subspace, i.e. the completion of $\lambda \mu X$. This completes the proof of 4.4 .

4.5. If $\mu X$ is locally fine, so are $n \mu X$ and $e \mu X$. Every precompact space $\mu X$ is locally fine.

Proof. The first part of the statement is obvious, since the cardinal number of a covering $\left\{U_{\alpha} \cap W_{\beta}^{\alpha}\right\}$ is the sum of the powers of the coverings $\left\{W_{\beta}^{\alpha}\right\}$ for each $\alpha$. The second part is obvious either by a direct use of the intersection axiom $\left(U_{\mathrm{ii}}\right)$ or from the facts that compact spaces, and subspaces of locally fine spaces, are locally fine.

We shall need three formulas: (i) $\lambda n \lambda=n \lambda$; (ii) $n \lambda n=\lambda n$; (iii) $c \pi c=\pi c$. (i) follows from 4.5. The proof of (ii) is a routine induction on the successive derivatives $\mu^{(\alpha)}$. The proof of (iii) is a routine application of Morita's Lemma (given in $\S 3$ ).

The functors $\lambda$ and $e$ do not commute. We leave it to the reader to verify this in the following example: $X$ is topologically an uncountable sum of twopoint discrete spaces $P_{\alpha}$; a covering is uniform provided that all but countably many $P_{\alpha}$ are each contained in one set $U_{\alpha}$ of the covering. We also note that $\lambda$ does not commute with $f, c$, or $s$. (As it happens, Example B proves all three parts of this statement.) We cannot prove a corresponding result for $n$, since it is consistent with the axioms for set theory that $n$ is the identity functor. We know of no further commutativity relations among the functors 
we have introduced; but it is worth mentioning again the unsolved problem whether $\pi$ and $e$ commute.

The functor $\lambda$ does not preserve quotients. We leave it to the reader to work out the details in the following example. Let $\mu X$ be the space of Example B. Let $f$ be the function on $\mu X$ into $m R$ which is defined by $f(0)=0$ and, for $x=\left(x_{i}\right), x_{n}=c \neq 0, f(x)=c(1-1 / n)$. Clearly $f$ is uniformly continuous. Let $R$ be the uniform relation induced by $f$. One can show that $\mu X / R$ is precompact (and hence locally fine), but $\lambda \mu X / R$ is not precompact.

Theorem 4.6. Every Cauchy filter in $e \lambda \mu$ is Cauchy in $n \lambda \mu$ (and a fortiori, in $n \mu)$.

Proof. Let $D$ be a Cauchy filter in $e \lambda \mu$. Let $\left\{U_{n \alpha}\right\}$ be a $\sigma$-uniformly discrete covering in $n \lambda \mu=\lambda n \lambda \mu$, with each collection $\left\{U_{n \alpha}\right\}$ for fixed $n$ a uniformly discrete collection of nonmeasurable power. Let $V_{n}=U_{\alpha} U_{n \alpha}$. Then $\left\{V_{n}\right\}$ is in $e \lambda \mu$ and hence some $V_{n}$, say $V_{m}$, is in $D$. Hence $D$, restricted to the subspace $(n \lambda \mu) V_{m}^{\prime}=\nu Y$, is Cauchy in $e \nu Y$. Now $\nu Y$ is a nonmeasurable sum of subspaces $U_{m \alpha}$. In the complete atomic Boolean algebra of all unions of atoms $U_{m \alpha}$, the set of all elements which are members of $D$ is an ultrafilter closed under countable intersection and therefore contains an atom [18]. This means that some $U_{m \alpha}$ is in $D$. In view of $4.4, D$ is Cauchy in $n \lambda \mu$ and hence also in $n \mu$.

A uniformisable space $X$ such that $e a X$ is complete is called $e$-complete, or real-complete $\left({ }^{\mathfrak{5}}\right)$.

4.7. Corollary. For any uniformisable space $X$, the following statements are equivalent:

(a) The topological space $\pi a X$ is real-complete.

(b) Every space $Y$ which is homeomorphic with the completion of a space homeomorphic with $X$ is real-complete.

(c) $a X=n a X$.

(d) aX contains no uniformly discrete subspace of measurable power.

Proof. (c) and (d) are equivalent by 2.3. Evidently (b) implies (a). (a) implies (d); for if $Y$ is a measurable uniformly discrete set in $a X, Y$ is complete in $a X$ and closed in $\pi a X$, but for any Ulam measure $m$ on $Y$, the family $\{S \subset \pi a X \mid m(S \cap Y)=1\}$ is a nonconvergent filter which is Cauchy in the uniformity $e a$ on the topological space $\pi a X$. Finally, (c) implies (b). For let $\nu Y=\pi \mu X$. If $e a Y=e \lambda a Y$ is not complete then, by 4.6 , neither is $n a Y$; a fortiori $n \nu Y=n \pi \mu X$ is not complete. But, as noted in $\S 3, n \mu$ is the l.u.b. of

(5) To read the growing literature on these spaces the following glossary is unfortunately necessary. They were introduced by Hewitt [6] under the name of Q-spaces. Shirota [14] proved a number of theorems, in particular that these are precisely the closed subspaces of products of real lines, and called them e-complete spaces. The term real-compact has been introduced by Gillman and real-complete by Jerison. In the book in preparation by Gillman and Jerison they will (at last report) be called realcompact (no hyphen). 
$n a$ and $\mu$, which in this case is $\mu$. That is, $\mu$ has a basis of nonmeasurable coverings; therefore so does $\pi \mu, n \pi \mu X=\pi \mu X$, and we have a contradiction.

Next we recall Morita's Theorem [11] that the Lindelöf property implies the star-finite property; i.e. if every open covering has a countable subcovering (as in a separable metric space) then every open covering has a starfinite refinement. Applying 3.1 and 4.2 we have at once

4.8. Every countable uniform covering of a locally fine space has a starfinite countable uniform refinement. Hence the functors $s, e, \lambda$, satisfy $s \lambda=e \lambda$.

4.9. Every countable star-finite uniform covering of any uniform space has a refinement of the form $\left\{U_{i} \cap W_{j}^{t}\right\}$, where $\left\{U_{i}\right\}$ and each $w^{i}=\left\{W_{j}^{i}\right\}$ are uniform coverings whose nerves are uniformly equivalent to subspaces of Euclidean spaces.

Proof. Let $\left\{V_{i}\right\}$ be a countable star-finite uniform covering. Define $i \equiv j$ to mean that $V_{i}$ and $V_{j}$ are joined by a finite chain of overlapping sets $V_{k}$. " $\equiv$ " is an equivalence relation with equivalence classes $C_{n}$. For each $n$ choose a set $V_{i}$ in $C_{n}$ and call it $U_{0}^{n}$. Having defined $U_{m}^{n}$, let $U_{m+1}^{n}$ be the union of all $V_{i}$ meeting $U_{m}^{n}$ but not meeting $U_{k}^{n}$ for $k<m$. Then $\left\{U_{m}^{n}\right\}$ is a uniform covering. Its nerve is simply a countable collection of line segments and rays, and thus a subspace of the plane. For each $m$ and $n$, since $\left\{V_{i}\right\}$ is star-finite, $U_{m}^{n}$ is a union of finitely many sets $V_{i}$ and meets only finitely many more sets $V_{i}$. Therefore, there is a finite uniform covering $w(m, n)$ such that its intersection with the set $U_{m}^{n}$ is finer than $\left\{V_{i}\right\}$. The nerve of $w(m, n)$ is a finite polyhedron and hence is homeomorphic to a subspace of some Euclidean space; since it is compact, any homeomorphism is a uniform equivalence.

We are now ready to complete the proof that $\pi$ and $c$ commute on locally fine spaces which have no uniformly discrete subspaces of measurable power. The credit for the last step is due to Richard Arens, who in 1955 suggested a construction of suitable real-valued functions which turn out to be uniformly continuous on $\mu^{(3)} X$ in general. However, we can now apply the result 1.6 of [8] noted in $\$ 3$, for a quicker proof of

\section{THEOREM 4.10. $c \pi \lambda n=\pi c \lambda n$.}

Proof. From 4.8, 4.9, and the lemma just mentioned we see that a Cauchy filter in $c \lambda \mu X$ is always Cauchy in $e \lambda \mu X$; using 4.6, we have an identification of the Cauchy filters in $c \lambda \mu X$ and in $n \lambda \mu X$. Replacing $\mu$ with $n \mu$ and using the relation $n \lambda n=\lambda n$, we have a one-to-one correspondence between the points of $\pi \lambda n \mu X$ and of $\pi c \lambda n \mu X$, leaving fixed all the points of $X$. Since the operators $\pi$ and $c$ do not change the real-valued uniformly continuous functions, hence $c \pi \lambda n \mu X=c \pi c \lambda n \mu X$. But $c \pi c=\pi c$, and the proof is complete.

We turn next to extension theorems. Let us first give a simplified proof of Katětov's extension theorem for bounded uniformly continuous functions [9]. The simplification is due jointly to Melvin Henriksen and the authors.

ThEOREM (KATĚTOV). A bounded uniformly continuous real-valued function on a subspace $\nu A$ of a uniform space $\mu X$ can be extended to be uniformly continu- 
ous over $\mu X$ (with the extension also bounded).

Proof. Let $h$ be a bounded uniformly continuous real-valued function on $\nu A$. Then $h$ is uniformly continuous on $f \nu A$, a subspace of $f \mu X$. Since $\beta \nu A$ is the closure $A^{-}$of $A$ in $\beta \mu X, h$ can be extended over $A^{-}$(necessarily bounded). Now by the Tietze extension theorem, $h$ can be extended to be a uniformly continuous function on the compact space $\beta \mu X$. Restricting $h$ to $X$ completes the proof.

On a locally fine space, a uniformly locally uniformly continuous function is uniformly continuous. In more detail,

4.11. If a function $f$ on $\mu X$ into $\nu Y$ is uniformly continuous on each of a family of subspaces of $\mu X$ which form a uniform covering, then $f$ is uniformly continuous from $\lambda \mu X$ into $\nu Y$. If $f$ is uniformly continuous on each member of a finite uniform covering of $\mu X$, then $f$ is uniformly continuous on $\mu X$.

Proof. The first statement is obvious. As to the second, let $\left\{V_{\alpha}\right\}$ be in $\nu$. Let $\left\{U_{i} \mid 1 \leqq i \leqq n\right\}$ be the finite uniform covering of $\mu X$ as given in the hypothesis. Then for each $i, U_{i} \cap f^{-1}\left(\left\{V_{\alpha}\right\}\right)=U_{i} \cap\left\{W_{\gamma}^{t}\right\}$ for some $\left\{W_{\gamma}^{t}\right\}$ in $\mu$. Then $\left\{O_{\beta}\right\}=\bigwedge_{i=1}^{n}\left\{W_{\gamma}^{t}\right\}$ is also in $\mu$. Since $\left\{U_{i}\right\} \wedge\left\{O_{\beta}\right\}$ is finer than $f^{-1}\left(\left\{V_{\alpha}\right\}\right)$, the proof is complete.

THEOREM 4.12. A uniformly continuous real-valued function on a subspace $\nu A$ of a locally fine space $\mu X$ has an extension which is uniformly continuous on $\mu X$. In particular, c $\lambda$ preserves subspaces.

Proof. Let $f$ mapping $\nu A$ into $m R$ be given. Let $V_{n}=f^{-1}(n-1, n+1)$ and $U_{n}=V_{n} \cup(X-A)$. Then $\left\{V_{n}\right\}$ is uniform on $\nu A$ and hence $\left\{U_{n}\right\}$ is uniform on $\mu X$. Let $\left\{W_{i}\right\}$ be a uniform star-refinement of $\left\{U_{n}\right\}$.

The function $f$ is defined on the subspace $A \cap W_{1}^{*}$ of the subspace $W_{1}^{*} \cap\left(A \cup W_{1}\right)$. Since $W_{1}^{*} \subset U_{n}$ for some $n$, so that $A \cap W_{1}^{*} \subset V_{n}, f$ is bounded on $A \cap W_{1}^{*}$. By Katětov's Theorem $f$ may be extended to a bounded uniformly continuous function $g_{1}$ and $W_{1}^{*} \cap\left(A \cup W_{1}\right)$ to $m R$. By 4.11 the function $f_{1}$ on $A \cup W_{1}$ defined by the values of $f$ and $g_{1}$ is uniformly continuous.

Having extended $f$ to $f_{n}$ on $A \cup \cup_{i=1}^{n} W_{i}$, the same argument yields an extension $f_{n+1}$ on $A \cup \cup_{i=1}^{n+1} W_{i}$. In this way we obtain, in the obvious way, a well-defined function $\bar{f}$, extending $f$ over all of $X$. On each $W_{i}, \bar{f}$ agrees with $f_{i}$. By 4.11, $\bar{f}$ is uniformly continuous on $\lambda \mu X$.

The following corollary is essentially known.

4.13. Corollary. A real-valued continuous function on a subspace $Y$ of a uniformisable space $X$ is continuously extensible over $X$ if and only if it is uniformly continuous in the uniformity induced on $Y$ as a subspace of $a X$.

5. More details. In this section we present some additional results and comment on unsolved problems connected with locally fine spaces. Some parts of the proofs are given at the end of the paper and some parts are left to the reader. 
THEOREM 5.1. For a uniform space $\mu X$, the derivative $\mu^{(1)}$ of $\mu$ is a uniformity provided (a) $\mu X$ is metric, (b) $\mu$ has a basis of $\sigma$-uniformly discrete coverings, or (c) $\mu X$ is uniformly locally compact. In case (b), $\mu^{(1)}$ also has a basis of $\sigma$-uniformly discrete coverings, and therefore all derivatives $\mu^{(\alpha)}$ are uniformities. In case (c) $\mu^{(1)} X=a X$.

The proof in the $\sigma$-uniformly discrete case depends on the same indexing scheme as in Theorem 1.2, which clearly works as well for $\sigma$-uniformly discrete coverings as for countable ones. If $\mu X$ is metric, then in the expression $\left\{U_{\alpha} \cap W_{\beta}^{\alpha}\right\}$ we may replace the coverings $w^{\alpha}=\left\{W_{\beta}^{\alpha}\right\}$ with the elements $w^{n}$ of a countable basis for $\mu$, and work the same indexing scheme with these auxiliary indices $n$. The uniformly locally compact case is quite routine.

5.2. For any uniform space $\mu X$, the derivative $\mu^{(1)}$ of $\mu$ is a regular uniformity in the sense of Morita [10]. Indeed, this is true whenever $\mu$ is a regular uniformity (a la Morita), and hence it is true for all $\mu^{(\alpha)}$.

This means that $\mu^{(1)}$ satisfies a certain weak version of the star-refinement axiom together with $\left(U_{\mathrm{i}}\right),\left(U_{\mathrm{ii}}\right),\left(U_{\mathrm{iv}}\right)$, and $\left(U_{\mathrm{v}}\right)$. With this axiom system, Morita has shown [10] that one can develop a substantial part of the ordinary theory of uniform spaces, particularly the theory of completion. However, Weil's Theorem does not hold; indeed, every regular space has a regular uniformity [10], so that there can be no assurance that continuous realvalued functions exist.

With respect to the two unsolved problems (i) is $\mu^{(1)}$ always a uniformity and (ii) is $e \pi=\pi e$, the present authors cannot visualize the structure of a possible counterexample. With respect to the problem "is every locally fine space a subspace of a fine space" (i.e. of a space $a X$ ), the difficulty is in determining whether or not a given space is a subspace.

To illustrate our comments consider

EXAMPLE C. Let $E$ be the set of all finite sequences of zeros and ones, partially ordered as follows: $x<y$ if $x$ is an extension of $y$, i.e. $y$ is an initial section of $x$. Let $E$ carry the discrete topology; and let a covering $\left\{U_{\alpha}\right\}$ of $E$ be uniform if the union of all residual sets contained in the $U_{\alpha}$ is a cofinal (residual) subset of $E$.

Obviously $\mu E$ is a uniform space having a basis of countable partitions. We shall show that $\mu E$ is locally fine and complete; and obviously $\mu E$ is not $a E$. This is the simplest example we know of a complete locally fine space which is not fine. Even in this example we do not know whether or not $\mu E$ is a subspace of any space $a X$.

Proof. To see that $\mu E$ is locally fine, consider any $\left\{U_{\alpha} \cap W_{\beta}^{\alpha}\right\}$ in $\mu^{(1)}$. For any $x$ in $E$, there exist $y$ in $E$ and an index $\alpha$ such that $y \leqq x$ and all successors of $y$ are in $U_{\alpha}$. There exist $z \leqq y$ and an index $\beta$ such that all successors of $z$ are in $W_{\beta}^{\alpha}$. Since these successors are also in $U_{\alpha}$, it follows that $U_{\alpha} \cap W_{\beta}^{\alpha}$ contains a residual set which includes a successor of $x$. Since $x$ is arbitrary, $\left\{U_{\alpha} \cap W_{\beta}^{\alpha}\right\}$ is in $\mu$, as required. 
To see that $\mu E$ is complete, consider any filter $D$ in $E$. If $D$ is Cauchy, there is at most one point common to all its elements, and if there is one then clearly $D$ converges. Suppose then that $D$ has empty intersection. The complements of the elements of $D$ form a covering $\left\{U_{\alpha}\right\}$ of $E$. For any $p$ in $E$ let $A(p)$ be the set of all successors of $p$. For each $x$ in $E$, there exist $y$ and $z$ : in $A(x)$ such that $A(y) \cap A(z)$ is empty (that is, $E$ is everywhere branching, or antidirected). Since $A(y), A(z)$, and $E-A(y)-A(z)$ together constitute a uniform covering, one of them (and only one) is in the Cauchy filter $D$. Hence either $A(y)$ or $A(z)$ is in $\left\{U_{\alpha}\right\}$. Since $x$ is arbitrary, the residual sets in the $U_{\alpha}$ are cofinal and $\left\{U_{\alpha}\right\}$ is uniform. But $D$ contains the complement of each $U_{\alpha}$ and hence no $U_{\alpha}$ is in $D$, a contradiction. Therefore $\mu E$ is complete.

We now apply Example C.

5.3. Every uniformisable space which has a nonprecompact uniformity has at least $2^{2 \aleph_{0}}$ different (but possibly uniformly equivalent) locally fine uniformities. Every noncompact space which is complete in some uniformity is complete in at least $2^{\aleph_{0}}$ different (but possibly uniformly equivalent) locally fine uniformities.

The first part of $\mathbf{5 . 3}$ follows from the fact that, under the given hypothesis, there are at least $2^{2 \aleph_{0}}$ different complete spaces $a Y$ homeomorphically containing $X$-a result of Colmez [4]. The second part is gotten by taking an infinite uniformly discrete subspace of $a X$, and then $2^{\aleph_{0}}$ infinite subsets of this set such that the intersection of any two is finite; and finally taking the finest uniformity on $X$ which induces the structure of Example $\mathrm{C}$ on a specified (and indexed) subset.

In deriving Theorem 4.10 we used the connection between general uniformities and metric ones (3.1) to invoke two fairly deep properties of metric spaces, namely Stone's theorem that every open covering has a $\sigma$-discrete open refinement and Morita's theorem that in the separable case every open covering has a star-finite open refinement. We can, of course, give direct proofs of the corresponding results for locally fine uniform spaces (and also for the uniformly locally finite refinements involved in the derivation of 4.4). These proofs are as complicated as the original ones of Stone and Morita. One item of interest is that while we have not been able to reproduce Stone's construction with less than the rather formidable Lemma 4.1, Morita's construction needs no more than the operation $\left\{U_{\alpha} \cap W_{\beta}^{\alpha}\right\}$. Specifically,

5.4. Every countable uniform covering of a uniform space $\mu X$ has a starfinite countable refinement in $\mu^{(1)}$.

This is not hard to see by inspecting Morita's proof [11]. Note that 1.2 seems to be required for the construction.

We conclude with a description of locally fine metric spaces.

Theorem 5.5. A complete metric space $\mu X$ is locally fine (i.e., $\mu X=a X)$ if and only if both of the following conditions are satisfied:

(a) The set $C$ of all nonisolated points of $X$ is compact.

(b) Outside any neighborhood of $C$, distances are bounded away from zero. 
A metric space is locally fine if and only if

$\left(\mathrm{a}^{\prime}\right)$ the set of all nonisolated points is precompact, and

$\left(\mathrm{b}^{\prime}\right)$ in any complete set of isolated points, distances are bounded away from zero.

Theorem 5.6. For a uniformisable space $X, a X$ is metric if and only if $X$ is metrizable and the set of nonisolated points of $X$ is compact. $X$ has a locally fine metric uniformity if and only if $X$ is metrizable and the set of nonisolated points of $X$ is separable.

TheOREM 5.7. For a metric space $\mu X, \lambda \mu X$ is metric if and only if the set of nonisolated points of $\pi \mu X$ is compact.

The family likeness in these theorems is apparent. Let us include another curious member of the same family.

5.8. Let $\mu X$ be a metric space. A real-valued function $f$ is uniformly continuous on $\mu X$ into aR if and only if $\mu X$ has a uniform partition $\left\{U_{\alpha}\right\}$ such that on $U_{1}, f$ is bounded and uniformly continuous into $m R$, and on every other $U_{\alpha}$, $f$ is constant. In particular, if $X$ is connected, $f$ is bounded.

Proof of 5.1(a). Let $\mu X$ be a metric space and $\left\{U_{\alpha} \cap W_{\beta}^{\alpha}\right\}$ a typical covering in $\mu^{(1)}$. Choose a countable basis $\left(y^{n}\right)$ for $\mu$, with $y^{n+1}<^{*} y^{n}$ for each $n$; and for each $\alpha$ choose an index $n=n(\alpha)$ such that $y^{n}<\left\{W_{\beta}^{\alpha}\right\}$. Choose $\left\{V_{\gamma}\right\}$ $<^{* *}\left\{U_{\alpha}\right\}$ in $\mu$. For each $\gamma$ let $p(\gamma)$ be the least integer $i$ such that there is $\alpha$ such that $V_{\gamma}^{* *} \subset U_{\alpha}$ and $n(\alpha)=i$. Define the covering $z=\left\{V_{\gamma} \cap Y_{\theta}^{p}\right\}$ by intersecting each $V_{\gamma}$ with the covering $y^{p}$, where $p$ is $p(\gamma)+1$. Then $z<*\left\{U_{\alpha} \cap W_{\beta}^{\alpha}\right\}$, and specifically $\operatorname{St}\left(V_{\gamma} \cap Y_{\theta}^{p}, z\right)$ is contained in any set $U_{\alpha} \cap W_{\beta}^{\alpha}$ satisfying the following conditions. (1) $U_{\alpha}$ contains $V_{\gamma}^{*}$, and subject to this condition, $n(\alpha)=n$ is as small as possible. Clearly $n \leqq^{\alpha} p(\gamma)$, so that $\operatorname{St}\left(Y_{\theta}^{p}, y^{n+1}\right)$ is contained in some $W_{\beta}^{\alpha} ;(2)$ choose such a set $W_{\beta}^{\alpha}$. To prove the assertion, consider any $V_{\delta} \cap Y_{\xi}^{q}$ in $z$ which meets $V_{\gamma} \cap Y_{\theta}^{p}$. There exists a set $U_{\epsilon}$ such that $n(\epsilon)$ $=p(\delta)$ and $U_{\epsilon}$ contains $V_{\delta}^{* *}$. Since $V_{\gamma}$ meets $V_{\delta}$, hence $U_{\epsilon}$ contains $V_{\gamma}^{*}$. Therefore $n$, as chosen under $(1)$, is $\leqq p(\delta)$. Thus $Y_{\xi}^{q}$, meeting $Y_{\theta}^{p}$, is a subset of $W_{\beta}^{\alpha}$, and $V_{\delta} \cap Y_{\xi}^{q}$ is contained in $U_{\alpha} \cap W_{\beta}^{\alpha}$, as required.

The proof of 5.1(b) is quite similar except that, since the desired conclusion is stronger, all the apparatus of 1.2 is needed. We do not know if $\mu^{(2)}$ must be a uniformity when $\mu X$ is metric.

We omit the routine proofs of 5.2-5.4. We also omit the proof of 5.5; a proof of a stronger statement than the second part of 5.5 may be found in [8]. Similarly for the first part of 5.6, an immediate consequence of Theorem 3 of B. Levshenko, Mat. Sb. vol. 42 (84) (1957) p. 482.

Proof of 5.6 (second part). If $X$ has a locally fine metric uniformity, then by 5.5 the set of nonisolated points of $X$ has a precompact metric uniformity, and hence is separable. Conversely suppose that $X$ is metrizable with distance $d$ and suppose that the subspace $C$ of nonisolated points has a countable base $V_{i}$. We shall construct a distance function $e$ on $X$ satisfying $\left(\mathrm{a}^{\prime}\right)$ and $\left(\mathrm{b}^{\prime}\right)$ of 5.5 . 
The family $P$ of pairs $\left(V_{i}, V_{j}\right)$, where $V_{i}^{-} \subset V_{j}$ and $d\left(V_{i}, X-V_{j}\right)>0$ is countable. Arrange the elements of $P$ into an infinite sequence (repeating elements if necessary) $\left\{\left(U_{i}, W_{i}\right)\right\}$. For each $n$ let $e_{n}(x)=2^{-n} \min \left[d\left(x, U_{i}\right), 1\right]$. For $x$ and $y$ in $C$ let $e(x, y)=\left(\sum_{n}\left[e_{n}(x)-e_{n}(y)\right]^{2}\right)^{1 / 2}$. It is well known that the mapping of $C$ into the Hilbert cube, defined by

$$
h(x)=\left(e_{1}(x), e_{2}(x), \cdots, e_{n}(x), \cdots\right)
$$

is a homeomorphism. The metric $e$ on $C$ is obtained by measuring distance as in the Hilbert cube. Hence $e$ is consistent with the topology on $C$. Since $\left|d\left(x, U_{i}\right)-d\left(y, U_{i}\right)\right| \leqq d(x, y), e(x, y) \leqq d(x, y)$ for $x$ and $y$ in $C$. We now extend $e$ to all of $X$. For $x$ in $C$ and $y$ in $X-C$, let $e(x, y)=\inf e(x, z)+d(z, y) \mid z \in C$. For $x$ and $y$ in $X-C$ let $e(x, y)=\inf \{e(x, z)+e(z, y) \mid z \in C\}$.

To show that $e$ is a metric it is sufficient to show that $e$ satisfies the triangle inequality, i.e., $e(x, z) \leqq e(x, y)+e(y, z)$. Suppose that $x$ and $z$ are both in $C$. If $y$ is in $C$, then the inequality is clear. If $y$ is in $X-C$, then the inequality follows since $e \leqq d$ on $C$. Suppose that $x$ is in $C$ and $z$ is in $X-C$. If $y$ is in $C$, then the inequality follows from the triangle inequality in $C$. If $y$ is in $X-C$, then the inequality follows from the triangle inequality for $d$ on $X$ and $e$ on $C$, and the fact that $e \leqq d$ on $C$. Analogously for $x$ in $X-C$ and $z$ in $C$. For $x$ and $z$ both in $X-C$, the inequality is apparent.

Clearly $e$ preserves the isolation of each point in $X-C$. Since the Hilbert cube is precompact, so is $C$ under $e$. Since distance in $X-C$ is measured via $C$, if a sequence of pairs of points have distances approaching zero, the points approach $C$ and hence include a Cauchy sequence. Thus the conditions of 5.5 are satisfied.

Proof of 5.7. Suppose that $\lambda \mu X$ is metric. By 5.5, the set of nonisolated points of $\pi \lambda \mu X$ is compact. By 4.4, $\pi \lambda \mu X=\lambda \pi \mu X$. The result then follows since $\lambda \pi \mu X$ is homeomorphic with $\pi \mu X$.

Suppose that the set of nonisolated points of $\pi \mu X$ is compact. Let $Y$ be the complete metric space $\pi \mu X$. By 4.2, $\lambda \pi \mu X=a Y$. By 5.6, $a Y$ is metric. Since $a Y=\lambda \pi \mu X=\pi \lambda \mu X$, the subspace $\lambda \mu X$ is metric.

Proof of 5.8. The sufficiency of the given conditions is trivial. Conversely, suppose that $f$ is uniformly continuous on $\mu X$ into $a R$, and a fortiori into $m R$. Consider the coverings (not open) $v^{n}$ of $R$ consisting of the interval $(-n, n)$ and all single points $y,|y| \geqq n$. If any $f^{-1}\left(v^{n}\right)$ is uniform, then it is a partition satisfying the hypothesis. Suppose that no such $v^{n}$ exists. Then for each $n$ there exist $x_{n}$ and $z_{n}$ in $X$ such that $d\left(x_{n}, z_{n}\right)<1 / n,\left|f\left(x_{n}\right)\right| \geqq n$ and $f\left(x_{n}\right)$ $\neq f\left(z_{n}\right)$. By taking a subsequence if necessary we may suppose that $\left|f\left(x_{n+1}\right)\right|$ $>\left|f\left(x_{n}\right)\right|$. Let $\left\{W_{n}\right\}$ be a disjoint family of open neighborhoods of the points $f\left(x_{n}\right)$, such that $f\left(z_{n}\right)$ is not in $W_{n}$. Let $W_{0}$ be the complement of the set of all $f\left(x_{n}\right)$. Since $\left|f\left(x_{n}\right)\right|$ goes to infinity, $W_{0}$ is open. Then $\left\{W_{i}\right\}$ is uniform on $a R$. But $\left\{f^{-1}\left(W_{n}\right)\right\}$ is not uniform, since none of its elements contains any pair $\left\{x_{n}, z_{n}\right\}$. This is a contradiction, and the proof is complete. 


\section{REFERENCES}

1. R. Bing, ed., Summary of lectures and seminars, Summer Institute on Set Theoretic Topology, Madison, 1955.

2. N. Bourbaki, Topologie générale (Chapter I), Actualités Sci. Ind. no. 858, Paris, 1940; (Chapter IX), Actualités Sci. Ind. no. 1045, Paris, 1948.

3. E. Čech, On bicompact spaces, Ann. of Math. vol. 38 (1937) pp. 823-844.

4. J. Colmez, Sur les espaces précompacts, C. R. Acad. Sci. Paris vol. 233 (1951) pp. 15521553.

5. S. Eilenberg and S. MacLane, General theory of natural equivalences, Trans. Amer. Math. Soc. vol. 58 (1945) pp. 231-294.

6. E. Hewitt, Rings of real-valued continuous functions, I, Trans. Amer. Math. Soc. vol. 64 (1948) pp. 45-99.

7. W. Hurewicz and H. Wallman, Dimension theory, Princeton, 1941.

8. J. Isbell, Euclidean and weak uniformities, Pacific J. Math. vol. 8 (1958) pp. 67-86.

9. M. Katětov, On real-valued functions in topological spaces, Fund. Math. vol. 38 (1951) pp. 85-91.

10. K. Morita, On the simple extension of a space with respect to a uniformity, I, Proc. Japan Acad. vol. 27 (1951) pp. 65-72.

11. - Star-finite coverings and the star-finiteness property, Math. Japon. vol. 1 (1948) pp. 60-68.

12. J. Nagata, On topological completeness, J. Math. Soc. Japan vol. 2 (1950) pp. 44-47.

13. P. Samuel, Ultrafilters and compactifications, Trans. Amer. Math. Soc. vol. 64 (1948) pp. $100-132$.

14. T. Shirota, $A$ class of topological spaces, Osaka Math. J. vol. 4 (1952) pp. 23-40.

15. A. Stone, Paracompactness and product spaces, Bull. Amer. Math. Soc. vol. 54 (1948) pp. 977-982.

16. A. Tarski, Über unerreichbare Kardinalzahlen, Fund. Math. vol. 30 (1938) pp. 68-89.

17. J. Tukey, Convergence and uniformity in topology, Princeton, 1940.

18. S. Ulam, Zur Masstheorie in der allgemeinen Mengenlehre, Fund. Math. vol. 16 (1930) pp. $140-150$.

National Cash Register Company,

Hawthorne, California

UNIVERSITY OF WASHINGTON,

Seattle, Washington 